\title{
AMENDMENTS
}

\section{Author Correction: Impact of carbohydrates, fat and energy density on energy intake}

\author{
R. James Stubbs (iD
}

Correction to: Nature Medicine https://doi.org/10.1038/s41591-021-01241-9, published online 1 February 2021.

In the version of this article initially published, several values in the table in Fig. 1 were incorrect. The corrections are as follows: for row 7 (Amount of food eaten), in column 2 (Low-carbohydrate), 2,140 g/d is revised to 1,473 g/d, and in column 3 (Low-fat), ' 1,4730 ' g/d is revised to $2,140 \mathrm{~g} / \mathrm{d}$; for row 9 (Glucose), in column $2,94.3 \mathrm{mg} / \mathrm{dl}$ is revised to $81.3 \mathrm{mg} / \mathrm{dl}$, and in column $3,81.3 \mathrm{mg} / \mathrm{dl}$ is revised to 94.3 $\mathrm{mg} / \mathrm{dl}$; for row 10 ( $\beta$-hydroxy butyrate), in column $2,0.2 \mathrm{mM}$ is revised to $1.8 \mathrm{mM}$, and in column $3,1.8 \mathrm{mM}$ is revised to $0.2 \mathrm{mM}$; for row 12 (24 hour), in column 2,2,315 kcal/d is revised to $2,294 \mathrm{kcal} / \mathrm{d}$, and in column $3,2,149 \mathrm{kcal} / \mathrm{d}$ is revised to $2,141 \mathrm{kcal} / \mathrm{d}$; and for row 13 (Physical activity), in column 2, $394 \mathrm{kcal} / \mathrm{d}$ is revised to $397 \mathrm{kcal} / \mathrm{d}$, and in column 3, $390 \mathrm{kcal} / \mathrm{d}$ is revised to $393 \mathrm{kcal} / \mathrm{d}$. Also, the descriptions of the diets in the title for the Fig. 1 legend ('plant-based high-carbohydrate' and 'animal-based high-fat') were inconsistent with the table headers. The correct descriptions are 'plant-based low-fat' and 'animal-based low-carbohydrate. The errors have been corrected in the HTML and PDF versions of the article.

Published online: 17 March 2021

https://doi.org/10.1038/s41591-021-01305-w

(c) Springer Nature America, Inc. 2021

\section{Author Correction: Patients could share virtual medical appointments for better access to telemedicine}

Kamalini Ramdas (D) and Soumya Swaminathan

Correction to: Nature Medicine https://doi.org/10.1038/s41591-020-01187-4, published online 13 January 2021.

In the version of this article initially published, reference 3 (Stevens, J. A. et al. Aust. Fam. Physician 45, 425-429 (2016)) was incorrectly cited in the second sentence of the third paragraph ("SMAs have also been used in Australia, including for aboriginal populations ${ }^{3}$...."). This reference should be deleted as a citation in the text and in the reference list (with the remaining references renumbered accordingly). The following new reference should be added to the end of the reference list and should be cited in the first sentence of the final paragraph (“The WHO's Global Strategy on Digital Health ${ }^{15}$, which was adopted....”): 15. World Health Organization. https://www.who. int/docs/default-source/documents/gs4dhdaa2a9f352b0445bafbc79ca799dce4d.pdf?sfvrsn=f112ede5_68 (2020). The errors have been corrected in the HTML and PDF versions of the article.

Published online: 30 March 2021

https://doi.org/10.1038/s41591-021-01306-9

(C) Springer Nature America, Inc. 2021 